\title{
LOGUNOV, D.V. \& Y.M. MARUSIK (2000): Catalogue of the jumping spiders of northern Asia (Arachnida, Araneae, Salticidae).
}

299 Seiten; Moskau (KMK Sci. Press). Englisch. ISBN 5-87317-081-9.

Institute for Systematics and Ecology of Animals, Siberian Branch of the Russian Academy of Science. Editor: K.G. Mikhailov. Mit einem Vorwort von Peter Merrett (Br. arachnol. Soc.).

Preis: US \$ 25 (exkl. Porto) bzw. GB $£ 20$ (inkl. Porto).

Bestellung: dmitri.v.logunov@man.ac.uk, Dr. Dmitri V. Logunov, Manchester Museum, University of Manchester, Oxford Road, Manchester M13 9PL, UK (oder bei der Europäischen Arachnologen-Tagung in Ungarn im Juli 2002).

Der stabil gebundene Band, gedruckt auf Hochglanzpapier im A5-Format, wirkt schon auf den ersten Blick recht ansprechend. Der Katalog umfasst ein riesiges Areal: Rußland vom Ural bis zum Pazifik, die Mongolei, beide Teile Koreas, sowie den Norden von Kasachstan, China und Japan (Hokkaido) - das Gebiet ist also in erster Linie geografisch definiert. Ein Blick auf einen Atlas/Globus zeigt, dass es (bis auf das flache sumpfige Westsibirien) in weiten Teilen Mittel- und vor allem Hochgebirgsareale enthält. Dazu kommen natürlich noch Habitate an Küsten, Fluss- und Seeufern, Sümpfe, usw.

Dem (obligatorischen) historischen Abriss über die Arachnologie im Gebiet, der Vorstellung verschiedener biogeografischer Verbreitungsmuster und der Erläuterung des Aufbaus folgt der eigentliche Katalogteil. 216 Springspinnen-Arten aus 41 Gattungen sind enthalten. Wie P. Merrett im Vorwort schon hervorhebt, gibt diese für einen Katalog geringe Zahl die Möglichkeit mehr ins Detail zu gehen. Diese Möglichkeit wird vorbildlich genutzt. Dem taxonomischen Teil (valider Name, Synonyme, Zitate) folgen Notizen zur Gesamtverbreitung jeder Art (auch außerhalb des Katalog-Gebietes). Die einzelnen Funde jeder Art sind mit geografischen Koordinaten, einer politisch-geografischen Zuordnung und den konkreten 
Zitaten versehen - damit ist der Katalogteil mit den Karten (insgesamt 55 - jeweils mehrere Arten pro Karte) für jede Art in vorbildlicher Weise transparent und nachvollziehbar. Es folgen bei jeder Art Angaben zu den Habitaten (mit Zitaten), ggf. Notizen zu Fehlbestimmungen und zur Taxonomie sowie Zitate der Art in Checklisten und anderen Katalogen.

Abgeschlossen wird der Katalog von einer Auflistung der nomina dubia und nomina oblita (letzteren Begriff verwenden die Autoren für die zwei betroffenen Arten nicht korrekt - es sind ebenfalls nomina dubia), Fehlbestimmungen und fragwürdigen Nachweisen (auch die kritische Betrachtung vieler Literaturnachweise ist richtungsweisend!), dem Literaturverzeichnis (314 Quellen) und einem Index.

Leider weichen die Autoren in wenigen Fällen (überwiegend Jahreszahlen betreffend) unbegründet vom internationalen Konsens ab. Beispiele: alle „Clerck-Arten“" sind mit 1758 anstatt, wie dies arachnologisch Usus ist, mit 1757 bezeichnet; Ballus depressus (praeocc.) anstatt B. chalybeius, Philaeus chrysops Poda, 1776 statt 1761. Weiterhin werden wenige Transfers sowie Synonymisierungen vorgenommen (die meisten im abstract genannt - dies ist leider bei anderen Katalogen nicht immer der Fall). Der Katalog ist aber insgesamt wohl einer der fehlerärmsten, die ich kenne. Z.B. konnte ich nur eine fehlende Art entdecken: Phintella cavaleriei (Schenkel, 1963) (laut Platnicks Internet-Katalog für Korea bekannt).

Fazit: Für alle, die sich für Springspinnen im Besonderen, asiatische Spinnen im Allgemeinen, oder auch für die Verbreitung paläarktischer Arten in Asien interessieren, und nicht zuletzt für diejenigen, die einen zukunftsweisenden Spinnenkatalog sehen bzw. haben wollen, ist dieses Werk ein Muss. 